\title{
Capacidad y modalidad. Una discusión de Aristóteles, Metafísica 1047a24-29 y 1048a11-16 ${ }^{1}$ \\ Capacity and Modality. A Discussion of Aristotle, Metaphysics 1047a24-29 and 1048a11-16
}

\author{
Ricardo SALLES \\ Instituto de Investigaciones Filosóficas, Universidad Nacional Autónoma de México \\ rsalles@unam.mx
}

\begin{abstract}
RESUMEN: En este artículo, me ocupo de dos pasajes de Aristóteles en los primeros cinco capítulos de Metafísica $\Theta$ estrechamente ligados entre sí (1047a24-29 y 1048a11-16). Intento mostrar, a través de ellos, que para Aristóteles podemos ser capaces de llevar a cabo una acción que resulta ser imposible en una situación dada. Esta tesis complementa otra igualmente importante, según la cual puede ser posible que realicemos una acción que, sin embargo, somos incapaces de realizar. Ambas tesis revelan que para Aristóteles los conceptos de capacidad y posibilidad en los primeros cinco capítulos de Metafísica $\Theta$ son independientes entre sí. Pero si bien la segunda tesis ha sido objeto de estudio en el reciente comentario de Stephen Makin al libro $\Theta$, la primera tesis no ha recibido toda la atención que merece.
\end{abstract}

ABSTRACT: In this paper, I examine two closely related passages from the first half of Aristotle's Metaphysics $\Theta$ (1047a24-29 and 1048a11-16) and seek to demonstrate through them that, according to him, we may have the capacity for doing an action that happens to be impossible in a given situation. This thesis complements another equally important thesis, namely, that it may be possible for us to do an action that we are incapable of doing. The two theses evince that for Aristotle the concepts of capacity and possibility in the first five chapters of Metaphysics $\Theta$ are independent from each other. But although the latter thesis has been studied in some detail by Stephen Makin in his recent commentary on $\Theta$, the former thesis has not received all the attention it deserves.

${ }^{1}$ Una versión anterior de este trabajo fue dictada como conferencia plenaria en el XIV Congreso de la Federación Internacional de Estudios Clásicos, celebrado del 25 al 30 de agosto de 2014 en Burdeos, Francia. Estoy agradecido con los miembros de la Mesa Directiva de la FIEC, y especialmente con la Dra. Martha Patricia Irigoyen, por su amable invitación. Una versión más reciente fue presentada el 29 de enero de 2015 en el Seminario de Investigación en Filosofía Antigua y su Recepción, Instituto de Investigaciones Filosóficas. Quiero agradecer a todos sus participantes sus valiosas observaciones. Este trabajo forma parte de una investigación más amplia que se lleva a cabo con el apoyo de los proyectos PAPIT IN400914 y CONACYT CB 2013-221268, que inició durante una estancia sabática en el Institute for Advanced Study de Princeton, EE.UU., con el apoyo del fondo Willis F. Doney Membership Endowment y del programa PASPA de la Dirección General de Asuntos de Personal Académico de la Universidad Nacional Autónoma de México. 
Palabras Clave: posibilidad; metafísica; acción; Aristóteles.

KEYwORDS: Possibility; Metaphysics; action; Aristotle.

RECIBIDO: 7 de enero de 2015 - ACEPTADO: 29 de febrero de 2016.

DOI: 10.19130/iifl.nt.2016.33.2.708

\section{Introducción}

En un estudio relativamente reciente sobre el libro $\Theta$ de la Metafísica de Aristóteles, ${ }^{2}$ Stephen Makin ha sostenido que, al interior de este libro, dedicado principalmente a la importante distinción entre ser 'en potencia'

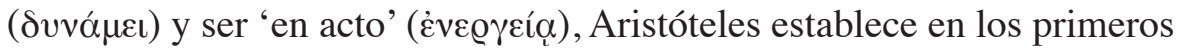
cinco capítulos una diferencia tajante entre los conceptos de capacidad y de posibilidad. Aunque Aristóteles usa un único término para referirse a

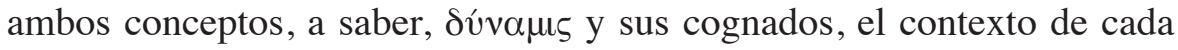
uso, argumenta Makin, deja claro cuándo, en esos capítulos, Aristóteles se refiere a una capacidad o a una posibilidad. La diferencia entre los dos conceptos estriba primordialmente en que la capacidad no es un concepto modal y, en particular, en que la posibilidad de que un agente lleve a cabo una acción no implica que ese agente sea capaz de llevarla a cabo. Por ejemplo, afirma Makin, puede darse el caso de que un estudiante principiante de arquitectura logre construir un puente y, por tanto, que sea posible que lo construya, sin que él tenga todavía, en sentido estricto, la capacidad de construir puentes, la cual es exclusiva del arquitecto o del ingeniero civil que ya domina plenamente su oficio. Esta tesis de la independencia entre la capacidad y la posibilidad ya había sido defendida por otros estudiosos de Aristóteles. ${ }^{3}$ Pero es gracias a Makin que se le ha asignado un lugar fundamental en la interpretación del libro $\Theta$ de la Metafísica. ${ }^{4}$ En este artículo, concuerdo enteramente con él y, de hecho, quiero demostrar que para Aristóteles la independencia entre los dos conceptos funciona en ambos sentidos. No sólo la posibilidad no implica la capacidad, como bien apunta Makin, sino que, como intentaré probar, la capacidad tampoco implica la posibilidad: para que un agente sea capaz de realizar una acción en una situación determinada, no se

${ }^{2}$ Cf. Makin 2006.

${ }^{3}$ Cf. Ross 1924, vol. 2, pp. 319-320; Beere 2009, pp. 49-50, 110-111 y 136-137, y Anagnostopoulos 2011, p. 401, n. 23.

${ }^{4}$ Cf. Makin 2006, pp. xxvii, 72-74, 112-118 y 122-124. 
necesita, piensa Aristóteles, que sea posible que la lleve a cabo. Para demostrar esto, me centraré en dos pasajes de $\Theta$ : el primero, 1047a24-29, del capítulo 3 y, el segundo, 1048a11-16, del capítulo 5. Al estudio del texto de estos pasajes dedico las secciones 2 y 3 de este artículo respectivamente. En la cuarta sección, me ocupo de una pregunta filosófica que surge naturalmente de la idea de que hay ciertas acciones imposibles que somos capaces de realizar: según Aristóteles ¿qué clase de imposibilidad está supuesta en estas acciones?

2. Met., $\Theta .3$ 1047a24-29

El texto del primer pasaje de $\Theta$ que deseo examinar figura en el capítulo 3 de ese libro y dice lo siguiente:

T1a: Met., $\Theta .3$ 1047a24-29

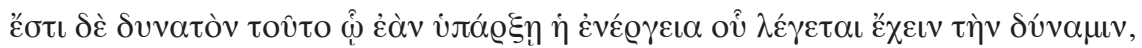

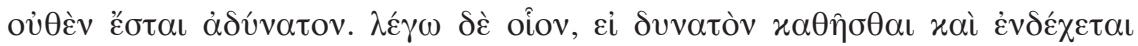

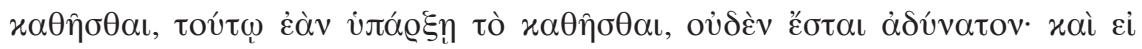

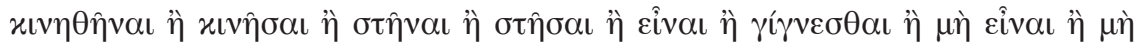

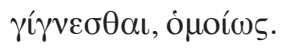

Si el acto de aquello de lo cual se dice que <el agente> es capaz se diera en él y no hubiera ninguna imposibilidad <en ello $>$, entonces esto $<$ sc. el acto de aquello de lo cual el agente es capaz $>$ es posible. Me refiero, por ejemplo, a que si es posible que <el agente> esté sentado, esto es, si admite estar sentado y estar sentado se da en él, entonces no habrá nada imposible <en ello $>$. De igual modo, si <para él es posible $>$ ser movido o mover, ponerse de pie o poner de pie, ser o generarse, o no ser o no generarse.

Creo que la idea clave de este difícil pasaje puede entenderse del modo siguiente. Supongamos que un agente $A$ es capaz de caminar y tratemos de imaginar una situación $S$ en la cual $A$ de hecho camina ('el acto de aquello de lo cual es capaz se da en él'). Si el hecho de que $A$ camine en $S$ no plantea ninguna imposibilidad, es decir, si la conjunción de esa acción con los hechos y los sucesos que se dan en $S$ no es imposible, entonces es posible que $A$ camine en $S$. De no ser así, es imposible que $A$ camine en $S$. En T1a, Aristóteles no indica las condiciones generales bajo las cuales es posible o imposible la conjunción de una acción con 
una serie de hechos y sucesos. Este será un tema del cual Aristóteles se ocupará en $\Theta .5$. En ese capítulo, él mencionará tres condiciones necesarias: una acción es posible en una situación determinada sólo si, en esa situación, (a) hay un paciente susceptible de recibir la acción, (b) no hay obstáculos externos que impidan su realización y (c) el agente tiene el deseo - que puede ser una elección, un anhelo o un apetito - de hacer la acción..$^{5}$ A menos de que se cumplan estas tres condiciones, la acción es imposible.

Antes de tratar este asunto, conviene notar que, según lo han advertido algunos estudiosos modernos de Aristóteles, ${ }^{6}$ los capítulos del libro primero de los $A$. Pr. dedicados a las deducciones modales contienen un análisis de la posibilidad parecido al de T1a:

T1b: A. Pr., 1.13 32a18-20

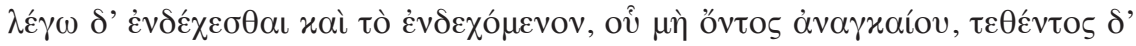

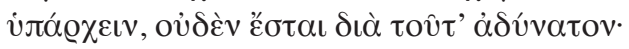

Por 'ser posible' y 'lo posible' quiero decir aquello que, no siendo necesario, si se postula que ocurre, no habrá nada imposible debido a ello.

Al igual que en T1a, lo posible se define aquí como aquello que, si se diera, no generaría una imposibilidad. Pero cabe señalar dos diferencias importantes. Una de ellas es que T1b se ocupa de aquello que es posible pero no necesario, es decir, de lo contingente. En cambio, T1a no se limita a este caso pues no excluye forzosamente que una acción necesaria sea 'posible'. En T1a no se exige que, para ser posible, una acción deba de ser no necesaria. La diferencia terminológica entre los dos pasajes y, en

5 No queda claro en $\Theta .5$ si, para Aristóteles, estas tres condiciones también son suficientes para que una acción sea posible. Si lo fueran, sería preciso distinguirlas de las condiciones suficientes para que la acción misma se realice, pues, de acuerdo con el propio Aristóteles, en términos generales las condiciones suficientes para la posibilidad de una acción tienen que ser distintas de las condiciones suficientes para la realización de la acción misma, pues de otro modo todo lo posible existiría en acto, es decir, no habría posibilidades no actualizadas. Las condiciones suficientes para la realización de una acción misma podrían estar dadas por la conjunción de las tres condiciones (a), (b) y (c) arriba mencionadas y de una cuarta condición sobre la cual Aristóteles hace hincapié en $\Theta .5$, a saber, que (d) el deseo de la acción es ‘decisivo' (xú olov). Cf. Met., 1048a10-12.

${ }^{6}$ Cf. Ross 1924, vol. 2, p. 245; Makin 2006, pp. 72-73, y Striker 2009, p. 127. 


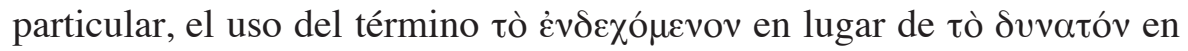
T1b podría tal vez leerse como una señal de esta diferencia conceptual. ${ }^{7}$ La otra diferencia importante radica en que T1a, al contrario de T1b, emplea la noción de capacidad para explicar qué es lo posible. En efecto, T1a es un pasaje que busca determinar, al interior del conjunto de acciones que un agente es capaz de hacer, aquellas que es posible que lleve a cabo. Es posible que lleve a cabo una acción en una situación determinada si, siendo capaz de llevarla a cabo, la conjunción de mi acción con los hechos y sucesos propios de esa situación no es imposible. Por consiguiente, a diferencia de T1b, T1a es prueba clara de que, para Aristóteles, puedo ser capaz de realizar una acción que resulta imposible.

T1a encierra una dificultad que no conviene pasar por alto. A primera vista, el propósito del pasaje es definir o explicar el concepto de lo posible mediante el concepto de lo no imposible. Pero dado que, de acuerdo con la lógica modal del propio Aristóteles, ${ }^{8}$ estos dos conceptos son equivalentes, si T1a fuera efectivamente una definición o explicación de lo posible, esta definición o explicación resultaría circular. La misma dificultad se plantea en T1b. Para resolver esta dificultad, algunos estudiosos han sostenido, correctamente a mi juicio, que el propósito de T1a y de T1b no es definir o explicar el concepto de lo posible, sino, más bien, formular un método, o prueba, para determinar, en casos difíciles, si una acción es posible o imposible en una situación determinada. David Ross, por ejemplo, al comentar sobre T1a, argumenta que "considered

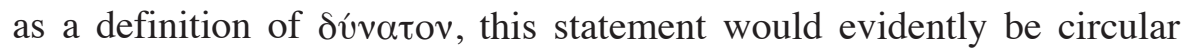
and therefore worthless. But it does not claim to be a definition. [...] It is a criterion for the determination of possibility in doubtful cases". $\mathrm{La}$ prueba consistiría en lo siguiente: si deseamos saber, pero no sabemos, si una acción que somos capaces de realizar es posible en una situación dada, determinemos si es posible la conjunción de esa acción con los hechos y sucesos que se dan en esa situación. Si es posible, entonces la acción misma es posible. De lo contrario, la acción es imposible. En

${ }^{7}$ Cf. Cat., 13 22b29-23a20 con comentario en Ackrill 1963, pp. 151-153, y EE, 2.6 1222b41-1223a9.

${ }^{8}$ Cf. int., 13 22a34-35: "pues la negación de lo imposible se sigue de lo posible y su afirmación de su negación" (<ảxo

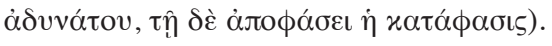

${ }^{9}$ Cf. Ross 1924, vol. 2, p. 245. Cf. Bonitz 1870, p. 387; Tricot 1981, p. 492, n. 1; Makin 2006, pp. 72-79, y Beere 2009, pp. 119-124. 
esta prueba, no se define ni explica el concepto mismo de lo posible. Al contrario, la prueba parte de que ya sabemos el significado de lo posible y se limita a establecer un procedimiento para determinar si, dado el significado de este concepto, una acción que somos capaces de realizar es posible en una situación dada. El procedimiento, como señalamos, consiste en considerar cada uno de los hechos y sucesos relevantes que se dan en una situación determinada para probar que el conjunto de todos ellos es consistente con que la acción ocurra. Un supuesto de esta prueba, desde luego, es que es más fácil determinar si una acción es consistente con un conjunto de hechos que determinar directamente si esa acción es posible. En cualquier caso, la pregunta de si T1a tiene como propósito definir y explicar el concepto de posibilidad o, al contrario, ofrecer solamente una prueba como la arriba descrita, no afecta mi argumento. Bajo cualquier lectura, este pasaje es evidencia de que, para Aristóteles, un agente puede ser capaz de realizar una acción que resulta imposible en una situación dada.

El objetivo del conjunto del capítulo al cual pertenece T1a, el capítulo 3 del libro $\Theta$, es echar abajo la concepción megárica de capacidad que a Aristóteles le parece absurda y defender una concepción alternativa a la megárica, que es precisamente la que él propone en T1a. Según los megáricos, sostiene Aristóteles, sólo somos capaces de llevar a cabo aquellas acciones que estamos realizando en este momento y sólo durante el tiempo en que las llevamos a cabo.

T1c: Met., $\Theta .31046 b 29-33$

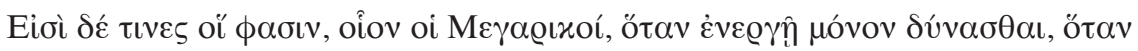

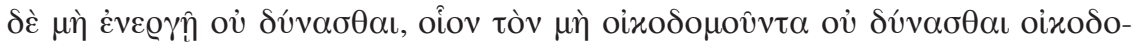

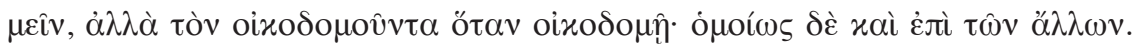

Y hay algunos que afirman, como los megáricos, que sólo se es capaz de actuar cuando se actúa y que, cuando no se actúa, no se es capaz, por ejemplo, que el que no está construyendo no es capaz de construir, sino sólo el que está construyendo cuando construye y de igual modo en los demás casos.

La tesis que aparece en T1a va más allá de la mera negación de esta tesis megárica. Ésta, según señalamos, se limita a aseverar que las únicas acciones que soy capaz de llevar a cabo son aquellas que estoy actualmente llevando a cabo. Para negar esto es suficiente demostrar que soy capaz de llevar a cabo una acción que no estoy actualmente llevando a 
cabo. No se necesita, además, argumentar que puedo ser capaz de llevar a cabo una acción que es imposible que lleve a cabo. El presente artículo no es el espacio adecuado para estudiar en detalle el lugar que ocupa T1a dentro de la estructura argumentativa de $\Theta .3$. Sin embargo, cabe notar que el conjunto de los argumentos que Aristóteles de hecho desarrolla en $\Theta .3$ contra los megáricos (1046b33-1047a24) busca probar, no la tesis fuerte que aparece en T1a de que puedo ser capaz de llevar a cabo una acción que me resulta imposible, sino la tesis más débil, pero suficiente para refutar a los megáricos, de que puedo ser capaz de llevar a cabo una acción que no estoy actualmente llevando a cabo. Si queremos encontrar los argumentos en los cuales se sustenta la tesis más fuerte de T1a, es preciso acudir a $\Theta .2$, el capítulo que precede a $\Theta .3$. En efecto, en $\Theta .2$ Aristóteles presenta una concepción epistémica de la capacidad para

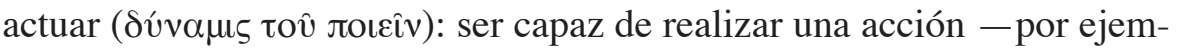
plo, curar- es simplemente conocer los medios necesarios y suficientes para realizarla. Dado que en eso consiste la capacidad para actuar, pero dado también que puedo tener ese conocimiento aun cuando me resulta imposible realizar la acción, se sigue que puedo ser capaz de realizar una acción aunque me resulte imposible realizarla. ${ }^{10}$

\section{Met., $\Theta .5$ 1048a11-16}

El segundo pasaje de $\Theta$ que deseo examinar proviene del capítulo 5, dedicado a determinar las condiciones necesarias y suficientes para que un agente dotado de cierta capacidad actúe conforme a ella. El texto es el siguiente:

T2a: Met., $\Theta .5$ 1048a11-16

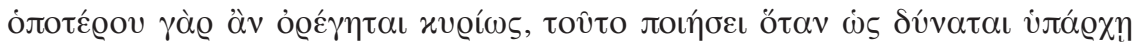

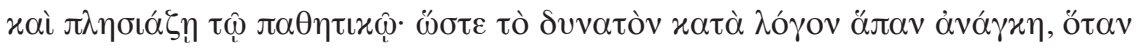

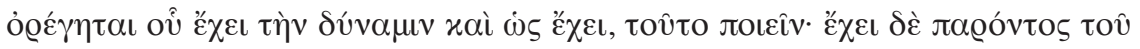

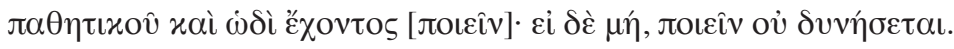

Pues, cualquier cosa que [el agente] desee de manera decisiva, la hará cada vez que sea capaz [de hacerla] y se aproxime al paciente. Por consiguiente, es necesario que todo aquel que sea capaz [de hacer algo] según la razón,

${ }^{10}$ Cf. Salles 2014. 
cada vez que desea aquello que tiene la capacidad de hacer, en cuanto la tiene, lo haga. Y tiene [tal capacidad] cuando lo pasivo está presente, y se halla en determinadas condiciones. De no ser así, no será capaz de actuar.

Las líneas sobre las cuales deseo detenerme son las últimas dos:

T2b: Met., $\Theta .5$ 1048a15-16

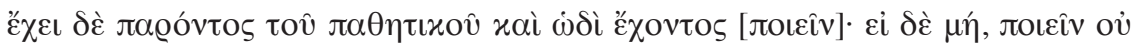

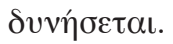

Y tiene [tal capacidad] cuando lo pasivo está presente, y se halla en determinadas condiciones. De no ser así, no será capaz de actuar.

Cito este pasaje conforme al texto de las ediciones de Ross y de Jaeger, el cual presenta dos problemas de sentido: (1) T2b parece establecer condiciones para que el agente posea una capacidad, lo cual resulta extraño habida cuenta de que el tema de $\Theta .5$ es, al contrario, el de cuáles son las condiciones para que un agente ejerza una capacidad que ya tiene; (2) las condiciones que se enuncian en T2b para la posesión de una capacidad parecen contradecir la teoría que el propio Aristóteles desarrolla en el libro $\Theta$ respecto de cómo se adquieren las capacidades. Makin ofrece una discusión pormenorizada de este problema en su comentario. ${ }^{11}$ La idea principal es que, de acuerdo con la teoría de las capacidades de Aristóteles al inicio del libro $\Theta$ y, en particular, en $\Theta .3$ 1046b36-1047a2, las capacidades racionales para actuar que no son innatas se adquieren por aprendizaje o entrenamiento y se pierden debido a factores como el olvido, una lesión física o el paso del tiempo. No es claro si esta lista de factores pretende ser exhaustiva, pero no cabe duda de que, para Aristóteles, un elemento común a todos ellos es que involucran un cambio en el agente. Si seguimos el texto de T2b en la edición de Ross y de Jaeger, sin embargo, en estas líneas Aristóteles afirmaría, por el contrario, que la mera presencia o ausencia de un paciente susceptible de recibir la acción del agente es suficiente para determinar si el agente posee o no una capacidad. En otras palabras, Aristóteles sostendría, contrariamente a lo que sostiene en $\Theta .3$ 1046b36-1047a2, que una capacidad puede adquirirse o perderse a través de un cambio puramente

${ }^{11}$ Cf. Makin 2006, pp. 112-118. Cf. Beere 2009, pp. 147-159. 
externo al agente, a saber, el cambio que consiste en que un paciente susceptible de recibir la acción se haga presente o ausente. Por ejemplo, imaginemos que María es un médico que tiene la capacidad de curar a su paciente, Juan. Imaginemos, además, que Juan es un viajero frecuente que, cada vez que sale de viaje, deja de poder recibir la acción de María. Pues bien, si siguiéramos el texto como lo editan Ross y Jaeger, habría que concluir que, para Aristóteles, cada vez que Juan saliera de viaje, María perdería la capacidad de curarlo y que, cada vez que él regresara, ella la recobraría. Pero esta conclusión es absurda si, como argumenta Aristóteles en $\Theta .3$ 1046b36-1047a2, la adquisición y la pérdida de capacidades racionales involucra cambios en el agente. Para evitar estos problemas, es preciso interpretar T2b en el sentido de que la ausencia de un paciente afecta, no la posesión de una capacidad, sino su ejercicio: su ausencia no implica una incapacidad, sino la imposibilidad de actuar conforme a una capacidad.

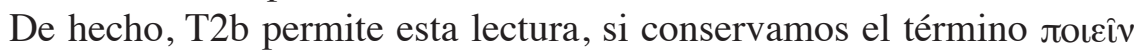
en la línea 1048a16 en vez de suprimirlo. Veamos el texto otra vez:

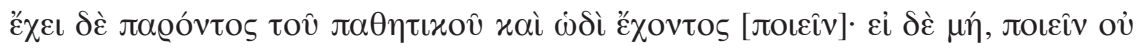

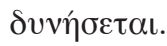

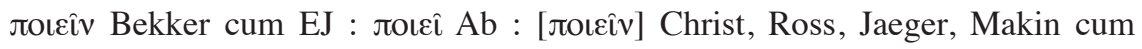
Alexander

Según lo apuntan las ediciones de Ross y de Jaeger, el primer Joเعîv de la línea 16 figura en los códices E y J (en Ab aparece лoเعî) y Bekker lo incluye, con toda razón, en el texto de su edición. Sin embargo, el término aparece omitido en Alejandro de Afrodisia y, a raíz de esto, lo suprime Christ seguido por Ross y Jaeger. De acuerdo con Ross, el

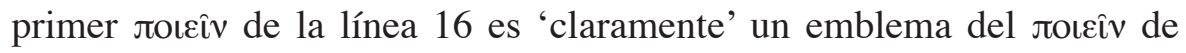

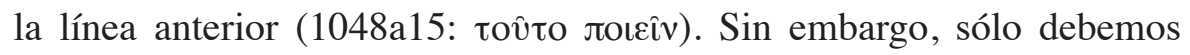
adoptar esta hipótesis si no es posible encontrar una lectura gramatical y filosóficamente plausible del texto que conserve el primer joเєîv de la

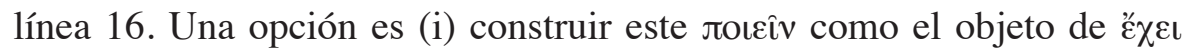

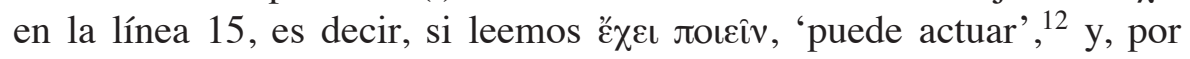

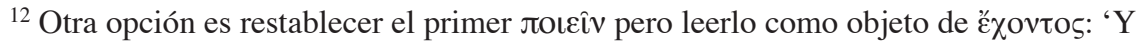
tiene [tal capacidad] cuando lo pasivo está presente y es posible que [el agente] actúe de este modo'. Una dificultad con esta lectura es que no hace sentido con la oración que
} 


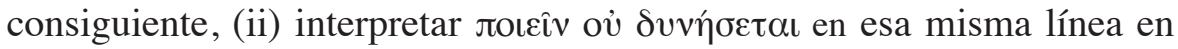
un sentido modal: 'actuar no será posible'. De este modo, el texto en su conjunto diría: 'y puede actuar si el paciente está presente y se halla en determinadas condiciones; de otro modo, actuar no será posible'. Bajo esta lectura, la imposibilidad de actuar no implica la incapacidad de actuar. Esto lo deja claro, en primer lugar, el contexto, pues el propósito de $\Theta .5$ es establecer las condiciones bajo las cuales un agente puede actuar conforme a una capacidad que posee $\mathrm{y}$, en segundo lugar, la gramática misma del pasaje, pues el sujeto de ع̌ $\chi \varepsilon \mathrm{r}$ en T2b es 'todo agente capaz de actuar conforme a la razón' en 1048a13.

Ahora bien, ¿puede leerse el primer Joเєîv de la línea 16 como objeto de "̌́ $\chi \varepsilon \iota$ en el sentido modal, es decir, para significar 'puede actuar'? Pienso que sí por dos razones. En primer lugar, la construcción de ě $\chi \varepsilon ı v$

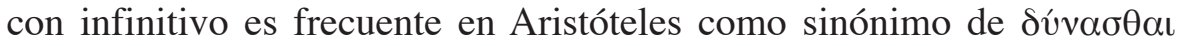
más infinitivo, como bien lo apunta Bonitz en el Index Aristotelicus. ${ }^{13} \mathrm{Y}$ esta construcción suele tener el significado modal de 'ser posible' como

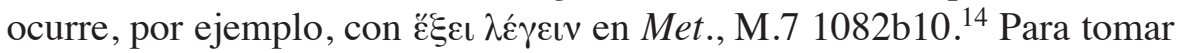
otro ejemplo, cabe citar el siguiente pasaje de la Política.

T2c: Pol., 1304b34-39

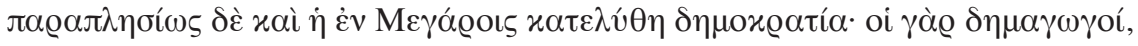

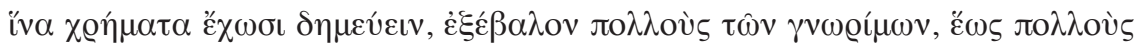

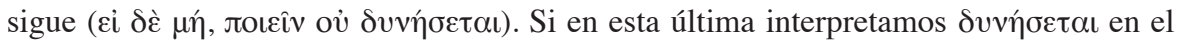
sentido de capacidad, el conjunto de las dos oraciones daría como resultado ' $Y$ tiene [tal capacidad] cuando lo pasivo está presente y es posible que [el agente] actúe de este modo. De no ser así, no será capaz de actuar'. Pero esto nos lleva de regreso al problema de que la posesión de una capacidad dependería de la presencia de un paciente, lo cual, como vimos, contradice lo que el propio Aristóteles afirma en $\Theta .3$ 1046b36-1047a2. En cambio, si interpretamos $\delta v v \eta ́ \sigma \varepsilon \tau \alpha \iota$ en el sentido de posibilidad, el conjunto de las dos oraciones daría como resultado 'Y tiene [tal capacidad] cuando lo pasivo está presente y es posible que [el agente] actúe de este modo. De no ser así, no será posible que actúe'. Pero el sentido de la segunda oración se vuelve sumamente oscuro: la primera oración establecería dos condiciones para que un agente tenga una determinada capacidad y esto implicaría que, si no se cumplen esas condiciones, el agente no poseería la capacidad; pero no implicaría que, si no se cumplen, la acción no es posible, como lo afirmaría la segunda oración si seguimos esta lectura.

${ }^{13}$ Cf. Bonitz 1870, p. 305b53-55.

${ }^{14}$ Para un estudio de los significados de $\delta u ́ v \alpha \sigma \theta \alpha \iota$ en $\Theta$, cf. Makin 2006, pp. xxiixxvii. 


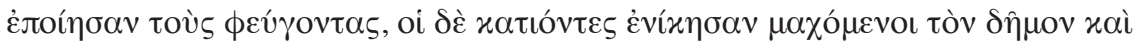

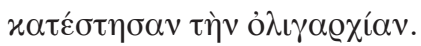

De un modo parecido se disolvió la democracia de los megarenses: los demagogos, en efecto, para poder confiscar sus bienes, expulsaron a muchos notables, hasta que fueron muchos los exiliados, y éstos, a su regreso, vencieron por las armas al pueblo y establecieron la oligarquía (traducción de Santa Cruz-Crespo con un cambio).

Ciertamente, la idea expuesta en T2c no es que los demagogos expulsaron a los notables para ser capaces de confiscar sus bienes, es decir, para saber cómo confiscarlos, que es como habría que entender el pasaje si interpretamos la construcción de $\varepsilon^{\prime} \chi \varepsilon v v$ con infinitivo de la línea

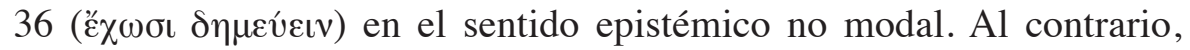
la idea central de T2c parece ser que, al saber que una condición necesaria para confiscar los bienes de los notables era expulsarlos de la ciudad, los demagogos los expulsaron para que fuera posible confiscar sus bienes, que es el modo en que podemos entender el pasaje si inter-

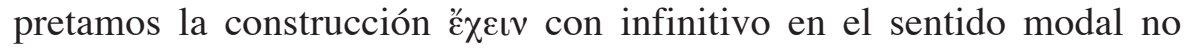
epistémico.

Podemos extraer la misma conclusión respecto de T2b si conservamos el primer лoเєîv de la línea 16: cuando Aristóteles afirma que el agente 'puede actuar si el paciente está presente', lo que quiere decir no es que la presencia del paciente le permite saber cómo realizar la acción (lectura epistémica no modal), sino que, gracias a la presencia del paciente, es modalmente posible que el agente actúe (lectura modal no epistémica).

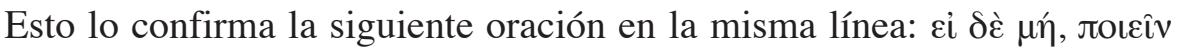

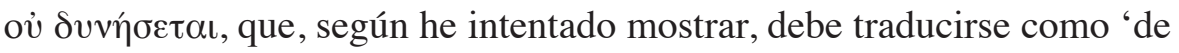
otro modo, actuar no será posible', y no como suele traducirse: 'de otro modo, no será capaz de actuar', ${ }^{15}$ si no queremos caer en el absurdo de que la posesión de una capacidad depende de la presencia de un paciente, absurdo que, como ya vimos, Aristóteles rechaza terminantemente al interior de $\Theta .3$ (1046b36-1047a2). Por consiguiente, cabe concluir que T2a prueba de forma adecuada que, para Aristóteles, un agente puede ser

${ }^{15}$ Una excepción es Tricot 1981, p. 497: “Et il peut agir, quand 1' objet de son activité est présent avec telle manière d' être; sinon, agir sera impossible"; Tricot sigue

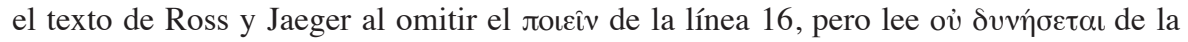
forma que yo propongo, a saber, en el sentido modal de imposibilidad. 
capaz de realizar una acción en una situación en la cual le es modalmente imposible realizarla.

Hasta aquí he intentado mostrar que los dos pasajes que nos propusimos analizar - $\Theta .3$ 1047a24-29 y $\Theta .5$ 1048a11-16- demuestran que, para Aristóteles, podemos tener la capacidad de realizar una acción que resulta ser imposible. Para terminar, deseo indagar la naturaleza de esta imposibilidad. ¿Qué clase de acciones imposibles somos capaces de realizar?

\section{4. ¿Qué clase de imposibilidades somos capaces de llevar a cabo?}

El único texto de $\Theta .1-5$ que permite entrever cuál sería la respuesta de Aristóteles a esta pregunta figura en $\Theta .5$, inmediatamente después de $\mathrm{T} 2 \mathrm{~b}$, en un pasaje que también se ocupa de las condiciones necesarias para el ejercicio de una capacidad.

T3a: $\Theta .5$ 1048a16-21

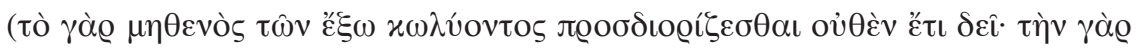

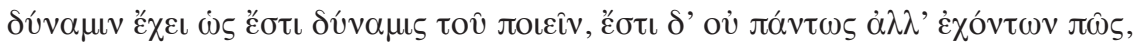

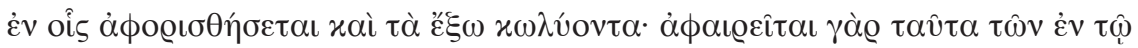

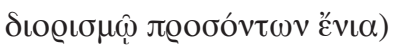

(en efecto, no es preciso añadir 'si nada lo impide', pues [el agente] tiene la capacidad en la medida en que es una capacidad para actuar y no [para actuar en general] sino siendo las cosas de cierto modo, en las cuales se excluyen los obstáculos externos, pues algunos elementos en la definición los suprimen).

Según T3a, cuando alguien determina las condiciones para el ejercicio de una capacidad, no hay necesidad de especificar 'siempre y cuando no hay obstáculos externos', es decir, no hay necesidad de especificar la condición (b). La razón, nos dice Aristóteles, es que la ausencia de obstáculos externos ya está presupuesta por la capacidad misma. Aristóteles expresa esta idea diciendo que la presencia de obstáculos externos

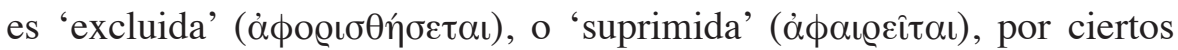

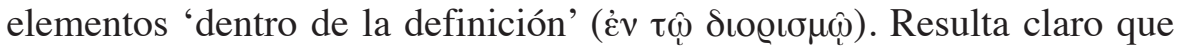
la definición a la que Aristóteles se refiere aquí es la del contenido de la capacidad, es decir, de la acción que la capacidad tiene como objeto. ${ }^{16}$

${ }^{16}$ Cf. Makin 2006, pp. 103-107 y 118-124, y Beere 2009, pp. 147-159. 
Para tomar un ejemplo, el contenido de mi capacidad para jugar tenis - el objeto de esa capacidad - es precisamente la acción de jugar tenis. La idea de Aristóteles es que la definición de esa acción incluye elementos que suprimen la presencia de obstáculos externos. No es sencillo entender por qué esto es así según Aristóteles. Si nos concentramos en el caso que acabo de ofrecer, una posible razón es que, para jugar tenis o cualquier otro deporte, tengo que llevar a cabo ciertos movimientos básicos que requieren la ausencia de obstáculos externos. Por ejemplo, estas reglas indican que, para jugar tenis, debo pegarle a la bola con una raqueta que sostengo con mis manos, lo cual implica que debo de sostener una raqueta con mis manos, lo cual, a su vez, implica que, para jugar tenis, ningún obstáculo externo debe impedirme sostener una raqueta con mis manos. En este sentido, la definición de la acción de jugar tenis involucra al menos un elemento - 'pegarle a la bola con una raqueta que sostengo con mis manos'- que excluye que una persona juegue tenis cuando se hallan presentes ciertos obstáculos externos.

Es preciso tomar nota de que, en Aristóteles, el concepto de definición tiene una carga modal. Según él, aquello que una definición no permite es imposible. Un pasaje importante al respecto es el que cito a continuación.

T3b: De Part. An., 640a33-b4

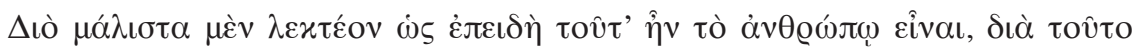

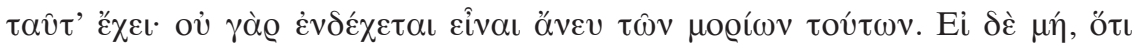

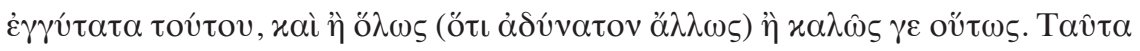

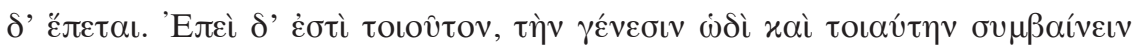

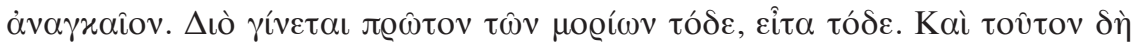

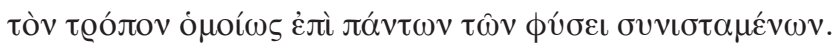

Por ello, debe decirse, sobre todo, que puesto que ésta es la esencia del ser humano, por eso tiene estas [partes]. Pues no es posible que exista sin estas partes. De no ser así, [debe decirse] lo más cercano a esto: o bien en general que [tiene estas partes] (porque es imposible que sea de otro modo), o bien en todo caso que es bueno [que las tenga]. Y esto se sigue [de lo anterior]. Pero puesto que [el ser humano] es de tal clase, su generación ocurre de este modo y resulta ser de tal tipo. Por ello, de las partes, se genera primero ésta y enseguida ésta. Y éste es el modo que se aplica por igual a todos los seres compuestos por naturaleza. 
Estas líneas revelan que, para Aristóteles, la esencia y, por ende, la definición de ser humano, están estrechamente ligadas con las condiciones necesarias para la existencia del ser humano y que estas condiciones, a su vez, se hallan también estrechamente ligadas con la imposibilidad de la existencia del ser humano cuando estas condiciones no se cumplen. Según la teoría de la definición de Aristóteles en la Metafísica, sólo las substancias tienen esencias y definiciones en un sentido estricto. ${ }^{17}$ Pero esta teoría no excluye ni (a) que haya otras cosas, distintas de las substancias, que también son susceptibles de definición en un sentido fuerte ni (b) que su definición tenga, al igual que la definición de las substancias, una carga modal, es decir, que su definición indique una serie de condiciones tales que es imposible que esas cosas se den cuando no se cumplen esas condiciones. Si es así, cabe interpretar la tesis central de T3a del modo siguiente: para toda acción $F$, la definición de $F$ excluye que $F$ ocurra en la presencia de ciertos obstáculos externos en el sentido de que, dada la definición de $F$, es imposible que $F$ ocurra cuando se dan esos obstáculos. La imposibilidad en cuestión se deriva directamente de la definición de la acción.

Para concluir, regresemos al punto de partida de este artículo, la tesis de que la capacidad no implica la posibilidad: hay acciones que somos capaces de realizar pero que es imposible que realicemos. En el apartado 2, vimos que no cabe duda de que Aristóteles acepta esta tesis y, en el presente apartado, intenté especificar qué tipo de imposibilidad está en juego cuando Aristóteles afirma que somos capaces de realizar acciones imposibles. Según lo apunté líneas atrás, se trata de una imposibilidad que se desprende de la definición misma de la acción. Puede darse el caso de que seamos capaces de realizar una acción $F$ en una situación dada aun cuando, dada la definición de $F$, sea imposible llevarla a cabo en esa situación. Para evitar confusiones al respecto, es preciso hacer una última aclaración. Es necesario distinguir entre dos tipos de imposibilidades que obedecen a una definición, a saber, (1) la imposibilidad de una acción que, por definición, es imposible en sí misma y (2) la imposibilidad de una acción cuya realización es, por definición, imposible en una situación dada. Por una acción 'por definición imposible en sí misma', entiendo una acción que se define por medio de un concepto que expresa una contradicción lógica como, por ejemplo, la acción de

${ }^{17}$ Cf. muy especialmente Met., Z.5 1031a1-2 y Z.13 1039a19-20. 
caminar y no caminar, la cual se define por medio de un concepto que expresa una contradicción como ocurre con el concepto de caminar y no caminar. En cambio, una acción como la de jugar tenis no es por definición imposible en sí misma, pues los conceptos que intervienen en su definición - las reglas del tenis - no expresan en sí mismos ninguna contradicción. Ahora bien, por una acción 'cuya realización es, por definición, imposible en una situación dada', entiendo una acción cuya definición implica que es imposible que esa acción se lleve a cabo en una situación dada. Por ejemplo, la acción de jugar tenis es una acción cuya realización es, por definición, imposible cuando el agente está atado de pies a cabeza porque la definición de esa acción implica que es imposible que un agente juegue tenis cuando está atado de pies a cabeza. Por lo tanto, si bien la acción de jugar tenis no es por definición imposible en sí misma, es una acción cuya realización es, por definición, imposible en una situación dada.

Esta distinción es de gran ayuda para entender correctamente la tesis de Aristóteles. Ciertamente, su postura no es que puedo tener la capacidad de realizar una acción por definición imposible en sí misma. De hecho, hay evidencia de que Aristóteles se opuso frontalmente a esta postura en el libro $\Theta .{ }^{18}$ Como he intentado mostrarlo en este último apartado, la postura que adopta Aristóteles en este libro y, en especial en los dos pasajes clave que aparecen en 1047a24-29 y 1048a11-16, es simplemente que puedo ser capaz de realizar una acción cuya realización es, por definición, imposible en una situación dada.

\section{BIBLIOGRAFÍA}

Anagnoustopoulos, A., "Senses of dunamis and the structure of Aristotle's Metaphysics $\Theta ”$, Phronesis, 56, 2011, pp. 388-425.

BeERE, J., Doing and Being. An interpretation of Aristotle's Metaphysics Theta, Oxford, Oxford University Press, 2009.

BonITZ, H., Index Aristotelicus, Berlin, G. Reimer, 1870.

JAEger, W., Aristotelis Metaphysica, Oxford, Oxford Classical Texts, 1957 [10a reimp. 1989].

Makin, S., Aristotle, Metaphysics Book $\Theta$, Oxford, Clarendon Press, 2006.

Ross, D., Aristotle's Metaphysics. A Revised Text with Introduction and Commentary, Oxford, Clarendon Press, 1924 [2a ed. 1953].

${ }^{18} \mathrm{Cf}$. en particular $\Theta .4$ 1047b3-14. 
SALLES, R., "Acciones alternativas y el origen platónico del concepto epistemológico de capacidad en Aristóteles", en L. Benítez, A. Velázquez (eds.), Tras las huellas de Platón en la filosofía moderna. De su simiente griega a la ilustración, México, DGAPA / UNAM, 2014, pp. 87-110.

TRICOT, J., Aristote, Métaphysique, Paris, Vrin, 1981. 